\title{
Efecto de algunas variables sobre la preñez de vaquillonas post-inseminación artificial a tiempo fijo
}

\author{
Erbiti, F. ${ }^{3}$, Lissarrague, C. ${ }^{2}$; Cabodevila, J. ${ }^{1}$ Callejas, S.S. ${ }^{1}$ \\ ${ }^{1}$ Centro de Investigación Veterinaria de Tandil (CONICET-ICPBA), Fac. Cs. Vet. Univ. Nac. Centro Prov. \\ Bs. As., Tandil, Argentina, Tel. 0249-4385850. ${ }^{2}$ Actividad privada, Tandil. ${ }^{3}$ Tesinista. \\ E-mail: callejas@vet.unicen.edu.ar
}

\begin{abstract}
Resumen
Erbiti, F.; Lissarrague, C.; Cabodevila, J.; Callejas, S.S.: Efecto de algunas variables sobre la preñez de vaquillonas post-inseminación artificial a tiempo fijo. Rev. vet. 29: 1, 35-39, 2018. El objetivo del presente trabajo fue evaluar el efecto del tratamiento de control del ciclo estral (Co-Synch: 5 días; J-Synch o Dispositivos 7 días), del estado reproductivo (cíclico o anestro superficial) y de la gonadotrofina coriónica equina (eCG) sobre el porcentaje de preñez post-inseminación artificial a tiempo fijo (IATF). Se utilizaron vaquillonas Angus de 20-22 meses $(\mathrm{n}=336)$, que fueron distribuidas a: Co-Synch 5 días (el día 0 se colocó un dispositivo intravaginal DISP $(0,558 \mathrm{~g}$ progesterona más $10,5 \mu \mathrm{g}$ de acetato de buserelina). El día 5 se retiró el DISP y se inyectó $0,150 \mathrm{mg}$ de D-Cloprostenol y aleatoriamente recibieron o no 300 UI de eCG. A las 56 h post DISP se realizó IATF más administración de 10,5 mg de acetato de buserelina. J-Synch: el día 0 se colocó DISP más $2 \mathrm{mg}$ de benzoato de estradiol. El día 6 se retiró el DISP y se inyectó $0,150 \mathrm{mg}$ de D-Cloprostenol; y aleatoriamente recibieron o no $300 \mathrm{UI}$ de eCG. A las $72 \mathrm{~h}$ post DISP se realizó IATF más $10,5 \mathrm{mg}$ de acetato de buserelina. Dispositivo 7 días: el día 0 se colocó DISP más $2 \mathrm{mg}$ de benzoato de estradiol. El día 7 se retiró el DISP y se inyectaron $0,150 \mathrm{mg}$ de D-Cloprostenol y $1 \mathrm{mg}$ de cipionato de estradiol; aleatoriamente recibieron o no 300 UI de eCG. El diagnóstico de gestación se realizó a los 30 días post IATF. Se observaron efectos significativos $(\mathrm{p}<0,01)$ del tratamiento de control del ciclo estral sobre el porcentaje de preñez (Co-Synch 5 días: $38,1^{\mathrm{a}}, J$-Synch: 55, $5^{\mathrm{b}}$ y Dispositivo 7 días: $61,1^{\mathrm{b}}$ ), del estado reproductivo (cíclico: 58,7; anestro superficial: 42,2 ) y de la administración de eCG (Si: 59,2 y No: 43,7$)$. Se concluye que el tratamiento hormonal, el estado reproductivo y la administración de eCG afectan el porcentaje de preñez post IATF.
\end{abstract}

Palabras clave: vaquillonas, tratamiento hormonal, estado reproductivo, administración de eCG, preñez post IATF.

\begin{abstract}
Erbiti, F.; Lissarrague, C.; Cabodevila, J.; Callejas, S.S.: Effect of some variables on the pregnancy of Angus heifers post fixed timed artificial insemination. Rev. vet. 29: 1, $35-39,2018$. The objective of the present work was to evaluate the effect of treatment of estrus cycle control (Co-Synch 5 days; J-Synch or Device by 7 days), reproductive status (cyclic or anestrus) and equine chorionic gonadotropin (eCG) on pregnancy rate after fixed time artificial insemination (FTAI). Angus heifers, 20-22 age $(\mathrm{n}=336)$ were assigned to $\mathrm{Co}$ Synch 5 days: on day 0 intravaginal device IVD $0.558 \mathrm{~g}$ progesterone was inserted with an injection of $0.105 \mathrm{mg}$ of busereline acetate. On day five IVD were removed and injected with $0.150 \mathrm{mg}$ of D-Cloprostenol and assigned to injected o not with $300 \mathrm{IU}$ of eCG. FTAI was performed at $56 \mathrm{~h}$ after IVD removal and all cows were administered $10.5 \mathrm{mg}$ of busereline acetate at these time. J-Synch: on day 0, IVD was inserted with an injection of $2 \mathrm{mg}$ of estradiol benzoate. On day 6, IVD was removed and injected with $0.150 \mathrm{mg}$ of D-Cloprostenol and assigned to injected or not with $300 \mathrm{IU}$ of eCG. Device by 7 days: on day 0, IVD was inserted with an injection of $2 \mathrm{mg}$ of estradiol benzoate. On day 7, IVD was removed and injected with $0.150 \mathrm{mg}$ of D-Cloprostenol and $1 \mathrm{mg}$ of estradiol cipionate, and assigned to injected o not with $300 \mathrm{IU}$ of eCG. Gestation diagnosis were performed at 30 days after FTAI. Treatment of estrus cycle control (Co-Synch 5 days: $38.1^{\mathrm{a}} ;$ J-Synch: $55.5^{\mathrm{b}}$ and Device by 7 days: $61.1^{\mathrm{b}}$ ), reproductive status (cyclic: $58.7 \%$; superficial anestrus: $42.2 \%$ ) and eCG administration (Yes: $59.2 \%$ and Not: $43.7 \%)$ affect pregnancy rate $(\mathrm{p}<0.01)$. In conclusion,
\end{abstract}


treatment of estrus cycle control, reproductive status and eCG administration, affect the pregnancy rate.

Key words: heifers, hormonal treatment, reproductive state, eCG administration, pregnancy post FTAI.

\section{INTRODUCCIÓN}

En aquellos rodeos de cría en los cuales se encuentran animales cíclicos y en anestro, el tratamiento utilizado para implementar programas de inseminación artificial a tiempo fijo (IATF) se basa en la colocación de dispositivos intravaginales con progesterona durante 7-8 días, combinado con la administración de sales de estrógenos y un agente luteolítico ${ }^{2}$. Este protocolo permite implementar la IATF entre las 48 y $54 \mathrm{~h}$ de retirados los dispositivos ${ }^{15}$.

En los últimos años, se ha trabajado en la reducción del tiempo en que permanece colocado el dispositivo en vagina (de 7-8 a 5-6 días) con la finalidad de prolongar la duración del proestro (a $72 \mathrm{~h})^{3}$. Esta innovación tiene por finalidad dar más tiempo al folículo dominante para que se desarrolle en un ambiente con bajos niveles de progesterona, que aumenten los niveles de estradiol durante ese ciclo y de progesterona en el subsiguiente; lo cual permitiría mejorar la fertilidad de la ovulación inducida ${ }^{4}$.

Por otro lado, se ha documentado que el estado reproductivo (anestro o cíclico) en el momento de iniciar un protocolo de control del ciclo estral, afecta el resultado de la IATF cuando se utiliza el protocolo tradicional ${ }^{6}$; en consecuencia, es de interés evaluar si esto ocurre cuando se utilizan tratamiento cortos.

Por último, la gonadotrofina coriónica equina (eCG) es una hormona que mejora las condiciones ováricas debido a su efecto estimulante sobre el desarrollo del folículo dominante, la tasa de ovulación y los niveles de progesterona que produce el cuerpo lúteo formado ${ }^{11}$; por lo tanto, se pretende evaluar si también tiene efecto en aquel tratamiento que prolonga el proestro.

El objetivo del presente trabajo fue evaluar el efecto del tratamiento de control del ciclo estral (Co-Synch 5 días; J-Synch o Dispositivos 7 días), del estado reproductivo (cíclico o anestro superficial) y del uso de la eCG sobre el porcentaje de preñez a la IATF.

\section{MATERIAL Y MÉTODOS}

Lugar, animales, alimentación. El trabajo se realizó en un establecimiento comercial ubicado en cercanías de Indio Rico, localidad del Partido de Coronel Pringles, Provincia de Buenos Aires, durante los meses de mayo y junio de 2015. Se trabajó sobre un rodeo de 574 vaquillonas Aberdeen Angus de 20-22 meses. Las vaquillonas habían sido destetadas en marzo de 2014, a los 6 a 7 meses de edad, con un peso promedio de $176 \mathrm{~kg}$. Durante su recría, etapa que se extendió hasta fines de febrero de 2015, ganaron en promedio $300 \mathrm{~g} /$ día. En marzo de ese
Tabla 1. Clasificación del estado reproductivo según las características de ovarios y útero.

\begin{tabular}{ll}
\hline clasificación & características \\
\hline cíclico & presencia de cuerpo lúteo con o sin folículo \\
& $>$ a $10 \mathrm{~mm}$. Tono uterino alto. \\
anestro & ausencia de cuerpo lúteo con presencia de \\
superficial & folículo $\geq$ a $10 \mathrm{~mm}$. Tono uterino medio a bajo. \\
anestro & ausencia de cuerpo lúteo con presencia de \\
profundo & folículos $<$ a $10 \mathrm{~mm}$. Tono uterino bajo. \\
\hline
\end{tabular}

año pesaban $285 \mathrm{~kg}$. Durante el otoño ocurrió una sequía en la región, donde la falta de precipitaciones hizo que el campo natural no produjera forraje en calidad y cantidad necesaria para cubrir los requerimientos de crecimiento de los animales. Cuando se realizó el primer encierre a para hacer la revisión pre-servicio (fines de mayo), las vaquillonas no habían ganado peso y se encontraban en un balance energético negativo.

Revisión ginecológica. Se realizó una revisión preservicio de los animales examinando el tracto genital mediante palpación trans-rectal del útero para determinar su tono (bajo, medio y alto) y ultrasonografía de los ovarios (Easiscan, BCF Technology; transductor lineal multifrecuencia de 4,5 a 8,5 MHz). Se determinó la estructura ovárica predominante (cuerpo lúteo-CL, folícu$\operatorname{los} \geq 0<$ a $10 \mathrm{~mm}$ de diámetro). Las vaquillonas fueron clasificadas según el tono uterino y la estructura ovárica en cíclicas y en anestro, superficial o profundo (Tabla 1).

Las vaquillonas seleccionadas para el experimento fueron 339 (59,1\% del rodeo) e incluyó aquéllas que tuvieron un CL (cíclicas) o un folículo $\geq 10 \mathrm{~mm}$ (anestro superficial). Un total de 161 vaquillonas $(28,1 \%)$ fueron descartadas por encontrarse en anestro profundo y el resto, por presentar diferentes alteraciones anatómicas (infantilismo, cérvix dobles). Durante el experimento una vaquillona murió y dos perdieron el dispositivo por lo cual fueron eliminadas del análisis de la información.

Tratamientos $y$ servicio. Las vaquillonas fueron distribuidas según un diseño factorial $2 \times 3 \times 2$ (efectos principales: estado reproductivo (cíclico o anestro superficial), tratamiento de control del ciclo estral (CoSynch 5 dias, J-Synch y Dispositivo 7 dias y administración o no de eCG) a los siguientes grupos:

Co-Synch 5 días. El día 0 se colocó un dispositivo intravaginal con $0,558 \mathrm{~g}$ de progesterona (Cronipres Monodosis, Biogénesis Bagó) más una inyección de $10,5 \mathrm{mg}$ de acetato de buserelina (Gonaxal, Biogénesis Bagó). El día 5 se retiró el dispositivo y se inyectó 0,150 mg de D-Cloprostenol (Enzaprost D-C, Biogénesis 
Tabla 2. Esquema de formación de grupos (diseño factorial $2 \times 3 \times 2$ ).

\begin{tabular}{llc}
\hline estado reproductivo & tratamientos de control ciclo estral & eCG \\
\hline \multirow{4}{*}{ cíclico } & Co-Synch 5 días & si \\
& J-Synch & si \\
& Dispositivo 7 días & no \\
& Co-Synch 5 días & ni \\
\hline \multirow{2}{*}{ anestro superficial } & J-Synch & si \\
& Dispositivo 7 días & si \\
& & no \\
& & si \\
\hline
\end{tabular}

Tabla 3. Efecto del tratamiento para controlar el ciclo estral, del estado reproductivo y de la administración de eCG sobre el porcentaje de preñez a la IATF.

\begin{tabular}{lc}
\hline efectos principales & $\%$ preñez \\
\hline tratamientos de control del ciclo estral & \\
Co-Synch 5 días & $38,1^{\mathrm{a}}(43 / 113)$ \\
J-Synch & $55,5^{\mathrm{b}}(61 / 110)$ \\
Dispositivo 7 días & $61,1^{\mathrm{b}}(69 / 113)$ \\
\hline estado reproductivo & \\
cíclico & $58,7^{\mathrm{a}}(111 / 189)$ \\
anestro superficial & $42,2^{\mathrm{b}}(62 / 147)$ \\
\hline administración de eCG & \\
SI & $59,2^{\mathrm{a}}(100 / 169)$ \\
NO & $43,7^{\mathrm{b}}(73 / 167)$ \\
\hline
\end{tabular}

${ }^{\mathrm{a}, \mathrm{b}}$ Valores con diferentes superíndices dentro de cada efecto principal difieren $\mathrm{p}<0,01$.

Bagó). En ese momento los animales fueron asignados aleatoriamente para recibir o no 300 UI de eCG (Novormon, Syntex). A las $56 \mathrm{~h}$ post retiro del dispositivo se realizó una IATF más la administración de $10,5 \mathrm{mg}$ de acetato de buserelina.

J-Synch. El día 0 se colocó un dispositivo intravaginal con $0,558 \mathrm{~g}$ de progesterona (Cronipres Monodosis, Biogénesis Bagó) más una inyección de $2 \mathrm{mg}$ de benzoato de estradiol. El día 6 se retiró el dispositivo y se inyectó 0,150 mg de D-Cloprostenol (Enzaprost D-C, Biogénesis Bagó). En ese momento los animales fueron asignados aleatoriamente para recibir o no $300 \mathrm{UI}$ de eCG. A las $72 \mathrm{~h}$ post retiro del dispositivo se realizó la IATF más la administración de $10,5 \mathrm{mg}$ de acetato de buserelina.

Dispositivo 7 días. El día 0 se colocó un dispositivo intravaginal con $0,558 \mathrm{~g}$ de progesterona (Cronipres Monodosis, Biogénesis Bagó) más una inyección de 2 mg de benzoato de estradiol. El día 7 se retiró el dispositivo y se inyectaron $0,150 \mathrm{mg}$ de D-Cloprostenol (Enzaprost D-C, Biogénesis Bagó) y $1 \mathrm{mg}$ de cipionato de estradiol (CRONI-CIP, Biogénesis Bagó). En ese momento los animales fueron asignados aleatoriamente para recibir o no $300 \mathrm{UI}$ de eCG. Los tres tratamientos descriptos precedentemente se distribuyeron según las vacas estuvieran cíclicas o en anestro superficial. El número total de grupos formados se describe en la Tabla 2.

Para el servicio se utilizó semen congelado/descongelado en pajuelas de $0,5 \mathrm{ml}$ proveniente de un toro de probada fertilidad. El diagnóstico de gestación se realizó por ecografía a los 30 días de realizada la IATF.

Análisis estadístico. Se evaluaron los efectos del tratamiento, del estado reproductivo, de la administración de eCG y de las interacciones correspondientes sobre el porcentaje de preñez a la IATF. Para realizar el análisis estadístico se utilizó el ProcCATMOD del paquete estadístico SAS ${ }^{14}$, fijando un nivel de confianza del $95 \%(\alpha=0,05)$.

\section{RESULTADOS}

Se observaron efectos significativos del tratamiento de control del ciclo estral, del estado reproductivo y de la administración de eCG sobre el porcentaje de preñez ( $<<0,01$; Tabla 3 ). Las interacciones dobles y triples no fueron significativas $(\mathrm{p}>0,05)$.

\section{DISCUSIÓN}

Con respecto a los tratamientos para controlar el ciclo estral, el protocolo Co-Synch 5 días fue el menos efectivo, preñándose el $38,1 \%$ de los animales tratados. Este hecho fue observado al utilizar este protocolo en vacas con ternero al pie, donde se preñó el $21,9 \%$ de las mismas; resultando menor que el de aquéllas tratadas con un dispositivo intravaginal con progesterona que permaneció en vagina durante 7 días $(45,7 \%, \mathrm{p}<0,05)^{8}$. Cabe señalar que tal efecto fue independiente de la estructura ovárica predominante (cuerpo lúteo, folículo $\geq$ $o<10 \mathrm{~mm}$ ); resultando similar al observado en el presente trabajo, en el que la respuesta fue independiente del estado reproductivo.

En otro trabajo realizado en vacas con cría en anestro ${ }^{7}$, el tratamiento Co-Synch 5 días también produjo un menor porcentaje de preñez $(26,8 \%)$ que el basado en la utilización de dispositivos intravaginales $(54,5 \%$, $\mathrm{p}<0,05)$. Sin embargo, el uso de la eCG revirtió el menor porcentaje de preñez $(46,3 \%, p>0,05)$; hecho no observado en el presente trabajo.

En otra investigación ${ }^{13}$ se utilizó el protocolo $\mathrm{Co}$ Synch 5 días en vaquillonas Holando Argentino obteniéndose un porcentaje de preñez similar al del tratamiento que utiliza el dispositivo con progesterona, colocado durante 7 días $(53,3 \%$ y 47,9\%, respectivamente; $p>0,05)$. En dicho trabajo, al momento de retirar el dispositivo, se administró una doble dosis del agente luteolítico, separadas por $12 \mathrm{~h}$; no así, en el presente trabajo en el que solo se utilizó una dosis.

Sin embargo, cuando se comparó el uso de 1 y 2 dosis del agente luteolítico en el protocolo Co-Synch 5 días en vaquillonas lecheras, no se encontraron diferencias en la regresión del cuerpo lúteo (86,9\% y 92,8\% 
respectivamente, $\mathrm{p}>0,05$ ) y en el porcentaje de preñez $(46,1 \% \text { y } 48,6 \% \text { respectivamente, } \mathrm{p}>0,05)^{12}$.

En coincidencia, se ha utilizado una sola dosis del agente luteolítico con resultado de preñez similar al de aquél en el que la duración del tratamiento fue de 7 días ${ }^{5}$. Por consiguiente, la administración de una dosis del agente luteolítico en el presente trabajo no sería la causa que explique el menor porcentaje de preñez registrado en el protocolo Co-Synch; no obstante, futuros trabajos deberán abordar este tema.

El porcentaje de preñez obtenido con el protocolo $J$-Synch resultó similar al del tratamiento tradicional (dispositivo por 7 días); coincidiendo esto con la bibliografía ${ }^{10}$. En dicho trabajo, el porcentaje de preñez fue $47,9 \%$ y $59,2 \%(\mathrm{p}>0,05)$ para los protocolos $J$-Synch y Dispositivo 7 dias, respectivamente.

En otro trabajo se informó un porcentaje de preñez menor, pero el mismo fue adjudicado a causas nutricionales (animales con pérdida de peso), que generarían condiciones en las vaquillonas que harían que el $J$ Synch tenga una menor respuesta comparada con la del tratamiento tradicional; de hecho este efecto no ocurrió cuando se revirtieron las condiciones nutricionales mencionadas ${ }^{3}$.

El estado reproductivo de los animales (cíclico y anestro superficial) afectó el porcentaje de preñez; observándose un mayor porcentaje en los animales cíclicos. Esta información es coincidente con lo observado en un trabajo similar donde se obtuvo un $56,3 \%$ de preñez en vacas cíclicas y $47,0 \%$ en las que se encontraban en anestro ${ }^{7}$.

En otra comunicación no se observaron diferencias en el porcentaje de preñez en vaquillonas de 15 meses de edad según se encontraran ciclando o en anestro ${ }^{9}$; no obstante, en dicho trabajo el porcentaje de animales con cuerpo lúteo fue del $71,5 \%$, superior al registrado en el presente trabajo $(33,6 \%)$, pudiendo estas diferencias ser la explicación a las discrepancias observadas. En apoyo de esta hipótesis, se ha observado un efecto del estado ovárico en vaquillonas con 24 meses de edad, con un porcentaje de animales con cuerpo lúteo del $23,7 \%{ }^{10}$, hecho similar al registrado en el presente trabajo.

La administración de eCG mejoró el porcentaje de preñez, pudiendo atribuirse este hecho al efecto positivo que tiene dicha hormona sobre el crecimiento del folículo dominante, el diámetro del folículo ovulatorio, el porcentaje de ovulación, el área del cuerpo lúteo y los niveles de progesterona ${ }^{11}$ que generan condiciones adecuadas para que se produzca la fecundación y se lleve a cabo la gestación.

Si bien los trabajos anteriores fueron realizado en vacas con cría; en una revisión publicada por investigadores brasileros ${ }^{1}$ se hace referencia a que vaquillonas Nelore tratadas con dispositivos con progesterona $\mathrm{y}$ eCG, tienen una mayor concentración de progesterona luego de ocurrida la ovulación y una mejora en el porcentaje de preñez en relación a las que no recibieron dicha hormona.
Se concluye que el tratamiento Co-Synch 5 días produce un efecto negativo sobre la eficiencia reproductiva, obteniéndose un menor porcentaje de preñez a la IATF comparado con el protocolo J-Synch y el Dispositivo 7 días. Éstos no difieren entre sí. El estado reproductivo (cíclico o anestro superficial), afecta el porcentaje de preñez y la administración de 300 UI de eCG en el momento de retirar el dispositivo intravaginal con progesterona, mejora el porcentaje de preñez.

\section{REFERENCIAS}

1. Baruselli PS, Sales JNS, Crepaldi GA, SáFilho MF. 2009. Uso de la eCG en biotecnologías reproductivas en bovinos. Resumen V Simposio Internacional de Reproducción Animal, Instit. Reprod. Animal, Córdoba, Argentina, p. 9.

2. Bó GA, Baruselli PS, Mapletoft RJ. 2013. Synchronization techniques to increase the utlization of artificial insemination in beef and dairy cattle. Anim Reprod 10: 137-142.

3. Bó GA, Mata JJ, Baruselli PS, Menchaca A. 2016. Alternative programs for synchronizing and resynchronizing ovulation in beef cattle. Theriogenology 86: 388-396.

4. Bridges GA, Mussard ML, Burke CR, Day ML. 2010. Influence of the length of proestrus on fertility and endocrine function in female cattle. Anim Reprod Sci 117: 208-215.

5. Colazo MG, Ambrose DJ. 2011. Neither duration of progesterone insert nor initial GnRH treatment affected pregnancy per timed-insemination in dairy heifers subjected to a Co-synch protocol. Theriogenology 76: 578-588.

6. Cutaia L, Veneranda G, Tríbulo R. Baruselli PS, Bó GA. 2003. Programas de inseminación artificial a tiempo fijo en rodeos de cría: Factores que lo afectan y resultados productivos. Resumen $V$ Simposio Internacional de Reproducción Animal, Córdoba, Argentina, p. 119-132.

7. Huguenine E et al. 2013. Efecto de la utilización de protocolos Co-Synch-5 días combinado o no con eCG en vacas con cría en anestro postparto. Resumen X Simposio Internacional de Reproducción Animal, Córdoba, Argentina, p. 313.

8. Iturralde $\mathbf{M}$ et al. 2013. Uso del protocolo Co-Synch + dispositivo de 5 días para implementar una IATF en vacas con cría. Resumen X Simposio Internacional de Reproducción Animal, Córdoba, Argentina, p. 315.

9. Lares S, Fernandez G, Formia N, Giovaninni R, Massara N, de la Sota RL. 2003. Eficacia de la utilización de un dispositivo intravaginal con progesterona monouso sobre la tasa de preñez en vaquillonas para carne de 15 meses. Resumen V Simposio Internacional de Reproducción Animal, Córdoba, Argentina, p. 405.

10. Menchaca A, López G. 2007. Tasa de preñez con la administración de GnRH 48 h luego de un tratamiento con estradiol, progesterona y eCG en vaquillonas de dos años. Resumen V Simposio Internacional de Reproducción Animal, Córdoba, Argentina, p. 259.

11. Núñez R, Castro T, García C, Bó G, Piaggio J, Menchaca A. 2014. Ovulatory response and luteal function after eCG administration at the end of a progesterone and estradiol based treatment in postpartum anestrous beef cattle. Anim Reprod Sci 146: 111-116. 
12. Rabaglino MB, Risco CA, Tratcher MJ, Kim IH, Santos JE, Thacher WW. 2010. Application of one injection of prostaglandin F2 $\alpha$ in the five-day Co-Synch + CIDR protocol for estrous synchronization and resynchronization of dairy heifers. J Dairy Sci 93: 1050-1058.

13. Ré M, Bó GA. 2013. Sincronización de la ovulación en vaquillonas lecheras utilizando tratamientos que prolongan el proestro. Resumen X Simposio Internacional de Reproducción Animal, Córdoba, Argentina, p. 324.
14. SAS 1998. User's guide, Version 6, Vol 2, $4^{\text {th }} \mathrm{ed}$, Cary MNC: SAS Inst. Inc., p. 846.

15. Zabala N, Repiso G, López A, Cutaia L. 2011. Efecto del momento de la IATF ( 48 vs. 54 h) en vacas cruza cebú con cría al pie tratadas con dispositivos con $0,5 \mathrm{~g}$ de progesterona y cipionato de estradiol sobre los porcentajes de preñez. Resumen $9^{\circ}$ Simposio Internacional de Reproducción Animal, Córdoba, Argentina, p. 338.

\section{SJR scrmaso Journal \& Country Rank}

\section{Powered by}

\section{Revista Veterinaria mantiene su índice de impacto}

Noticias de Scimago Research Group (Scimago Journals \& Country Ranks, Scopus-Elsevier) comunican que la publicación de nuestra casa, Revista Veterinaria, aumentó su índice de impacto. El índice SJR mide la influencia científica (impacto) del artículo de una revista, expresando cuán importante es el "artículo promedio" de la publicación en la discusión científica global (sistema Thomson Reuters).

Para nuestra revista, tal indicador había sido de 0,03 entre 2008 y 2011, aumentando a 0,05 en 2012, a 0,11 en 2013, a 0,108 en 2015 y a 0,100 en 2017, último período evaluado. Asimismo, surge para nuestra publicación un sostenido descenso del indicador que relaciona "citas versus autocitas", demostrando que los autores de los artículos están abandonando la práctica de citar sus propias publicaciones anteriores.

Por último, se advierte que según este portal, nuestra "Revista Veterinaria" continúa siendo la única publicación de esta rama de la ciencia que posee índice de impacto en Argentina. Para el resto del cono sur tal distinción recae en Brasil, Chile, Colombia y Venezuela. En revistas de veterinaria, a nivel mundial el mayor índice de impacto (2,45 puntos) recae en Annual Review of Animal Biosciences (Estados Unidos). 